\title{
Palisaded and Neutrophilic Granulomatous Dermatitis in a Patient with Dermatomyositis and Nasopharyngeal Cancer
}

\author{
Chii-Shyan Wang ${ }^{1}$, Hsuan-Hsiang Chen ${ }^{2}$ \\ ${ }^{1}$ Department of Dermatology, En Chu Kong Hospital, New Taipei City, Taiwan \\ ${ }^{2}$ Department of Dermatology, National Taiwan University Hospital and National Taiwan University College of Medicine, Taipei, Taiwan
}

Received: September 22, 2016 ; Accepted: September 20, 2016; Published: October 06, 2016

*Corresponding author: Hsuan-Hsiang Chen, MD, Department of Dermatology, National Taiwan University Hospital, 7,Chung-Shan South Road, Taipei, Taiwan, Tel: +886-2-2356-2141; Fax: +886-2-2393-4177; E-mail address: beauty101@gmail.com

\begin{abstract}
Palisaded and Neutrophilic Granulomatous Dermatitis (PNGD) is a rare form of neutrophilic dermatoses with granulomatous inflammation. It is a benign, inflammatory dermatosis with distinct histopathological features and varied clinical presentations. Associated systemic conditions include connective-tissue diseases and lympho proliferative disorders. Dermatomyositis associated PNGD was rarely reported before. Herein, a PNGD patient with dermatomyositis and nasopharyngeal cancer was presented. The clinical and pathological findings as well as mechanism of neutrophilic and granulomatous infiltrates in autoimmune connective tissue disease related skin lesions were also briefly discussed.
\end{abstract}

Keywords: Palisaded and Dermatitis; Dermatomyositis; Neutrophilic Granulomatous; Nasopharyngeal Cancer;

\section{Case Report}

A 42-year-old male patient went to our clinic in 2004, presented with erythematous patches and plaques on nasal base, nose and bilateral hands for 4 months. There was no oral ulcer, muscular weakness, or arthralgia. The clinical impression was tumid lupus erythematosus. The laboratory examination results revealed a positive antinuclear antibody (ANA: 1:1280, speckled type), positive anti-La antibody, and negative anti-Ro, anti-Sm/RNP, anti-Scl 70 antibodies. The complement C3 and C4, complete blood count, liver and renal profiles were all within normal limits. The skin biopsy and direct immune fluorescent examination (DIF) were not performed during that time. Topical steroids and oral hydroxychloroquine $200 \mathrm{mg}$ twice a day were prescribed. After 9 years of irregular follow up, this patient was found to have nasopharyngeal cancer and received radiotherapy. A repeated cutaneous physical examination revealed no Heliotrope sign, Gottron papules, periungual erythema, poikiloderma, or shawl sign. No muscular weakness was found either. The repeated laboratory examination for autoantibody confirmed positive anti-Ro and anti-La antibodies, and negative anti-Sm/RNP antibody. Six months later, he developed muscular weakness and elevated creatine phosphokinase (CPK: 2,266
IU/L). He was then diagnosed as dermatomyositis. After 1 year, multiple persistent itching round infiltrated erythematous plaques developed on the upper back and bilateral arms (Figure1 and 2). A skin biopsy was performed on the elevated plaque of the back and the sample was sent for histological examination. Microscopic examination showed perivascular interstitial mixedcell infiltrate, composed of neutrophils, histiocytes, lymphocytes, and nuclear dusts within the superficial and deep dermis (Figure 3 and 4 ). In some foci, the palisaded granuloma was

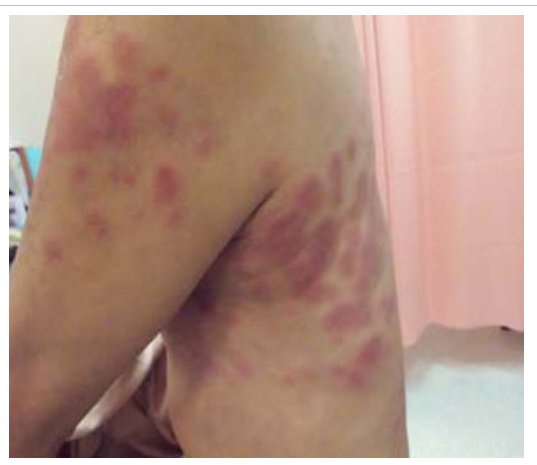

Figure 1: Multiple annular erythematous in durated plaques over upper back and bilateral arms.

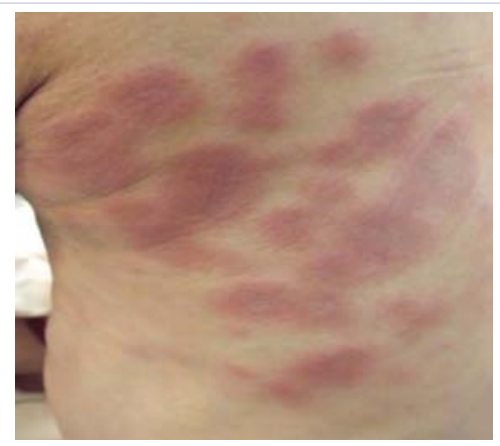

Figure 2: A closer view of multiple annular erythematous indurated plaques over upper back. 
found without vasculitis, features consistent with palisaded and neutrophilic granulomatous dermatitis. The absence of palisaded granulomatous inflammation with central necrobiosis and mucin deposition ruled out the possibility of granuloma annulare. The DIF of the skin biopsy showed only weak granular IgG and linear IgM deposition along dermo-epidermal junction, without definite diagnostic conclusion. The skin lesions improved after treatment with topical clobetasol proprionate ointment.

\section{Discussion}

Palisaded and Neutrophilic Granulomatous Dermatitis (PNGD) is a rare cutaneous manifestation, which is most commonly reported with rheumatoid arthritis [1]; however, it has also been associated with systemic lupus erythematosus [29], inflammatory bowel disease [10], and systemic sclerosis [11]. Other related diseases like Behcet disease, hepatitis, sarcoidosis, leukocytoclastic vasculitis has been reported, too [12-15]. To the best of our knowledge, PNGD associated with dermatomyositis has never been reported so far [16]. The clinical manifestations of PNGD include asymptomatic or intensely painful papules, nodules, linear subcutaneous in durated cordlike bands (the burning rope sign) [4], and plaques on multiple body sites. The histological examination typically shows a dense neutrophilic infiltrate with degenerated collagen, leukocytoclastic debris, and palisading granulomas, without vasculitis [3]. Chu et al proposed that the histological appearances of palisaded neutrophilic granulomatous dermatitis might vary from early (dense inflammatory infiltrates, composed of lymphocytes, neutrophils,

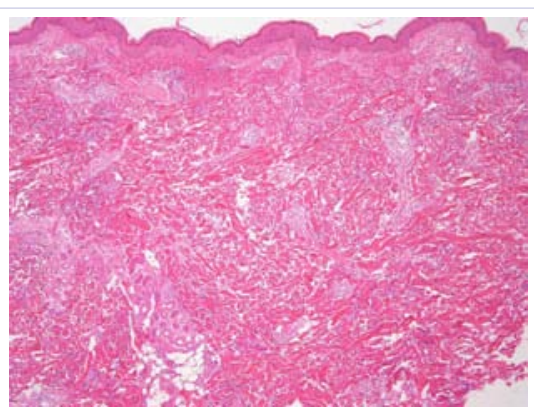

Figure 3: There was a perivascular interstitial mixed-cell infiltrate within the superficial and deep dermis. In some foci, the palisaded granuloma was found. (H \& E, original magnification $\times 40$ )

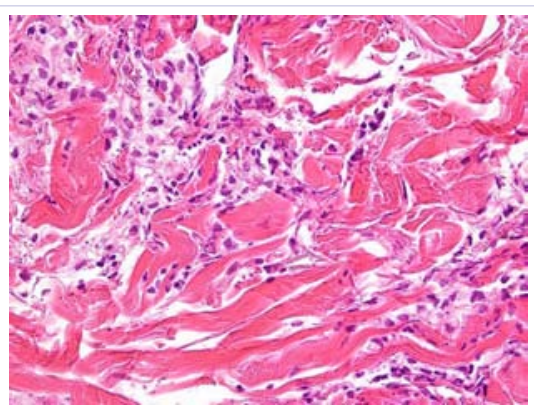

Figure 4: A closer view of the interstitial mixed-cell infiltrate composed of neutrophils, histiocytes, lymphocytes, and nuclear dusts. (H\&E, original magnification $\mathrm{x} 400$ ). histiocytes, and eosinophils) to late stages (palisading granulomas with fibrosis) of the disease [3]. The clinical and pathological presentations of our patient correlate with the previous findings in the literature.

The etiology of PNGD is unknown. Finan and Winkelmann observed IgM and C3 in small vessels by using DIF in 1983 [17]. They presumed that the cutaneous lesions were the result of immune complexes generated by underlying systemic diseases. Although the DIF of this patient did not show any deposition along the small vessels, there were still weak IgG and IgM depositions along dermo-epidermal junction, which might be due to the underlying dermatomyositis. The neutrophilic infiltrate in dermatomyositis related PNGD may be explained by the deposition of the immune complex and further activation of complement cascade. The adaptive immune system, particularly through the $\mathrm{T}$ helper lymphocytes (Th) 17-cell subset, may also secrete neutrophil-recruiting chemokines, such as IL-17A and G-CSF. Local synthesis of IL-17A by infiltrating Th17 cells in skin lesions of Autoimmune Connective Tissue Diseases (AICTDs) could lead to the presence of a nonspecific neutrophilic infiltrate in PNGD of AICTDs [18]. Neutrophils may also be over recruited in AICTD skin lesions because of an abnormal expression and/or activation of adhesion/migration molecules through the endothelial barrier of inflamed tissues [19]. Caproni et al proposed that the neutrophilic infiltrate described in specific skin lesions of dermatomyositis could be explained by increased expression of adhesion molecules, such as vascular cell adhesion molecule-1, intercellular adhesion molecule-1, and E-selections on endothelial cells [20]. Therefore, it is possible that the deposition of immune complexes in dermal vessels from underlying autoimmune disease activates the complement and Th17 lymphocytes, leading to over recruited aggregation of neutrophils and other inflammatory cells, causing degeneration and ischemia of collagen, followed by granulomatous reaction to this damaged collagen. According to Rosenbach and English [16], the unifying term "reactive granulomatous dermatitis" was proposed to encompass PNGD, interstitial granulomatous dermatitis, and interstitial granulomatous drug reaction, in order to emphasize these specific cutaneous granulomatous reaction patterns that occur in the setting of a systemic trigger.

In conclusion, we reported a rare association between PNGD and dermatomyositis. A new term, reactive granulomatous dermatitis, is suggested to describe these diverse clinical and pathological reaction patterns in response to some underlying systemic process.

\section{References}

1. Sangueza OP, Caudell MD, Mengesha YM, Davis LS, Barnes CJ, Griffin JE, et al. Palisaded neutrophilic granulomatous dermatitis in rheumatoid arthritis. J Am Acad Dermatol. 2002;47(2):251-257.

2. Germanas JP, Mehrabi D, Carder KR. Palisaded neutrophilic granulomatous dermatitis in a 12-year-old girl with systemic lupus erythematosus. J Am Acad Dermatol. 2006;55(2 Suppl):S60-62. DOI: 10.1016/j.jaad.2005.08.050

3. Chu P, Connolly MK, LeBoit PE. The histopathologic spectrum of palisaded neutrophilic and granulomatous dermatitis in patients with collagen vascular disease. Arch Dermatol. 1994;130(10):1278-1283. 
4. Gulati A, Paige D, Yaqoob M, Proby CM, Cerio R, Harwood CA. Palisaded neutrophilic granulomatous dermatitis associated with systemic lupus erythematosus presenting with the burning ropesign. J Am Acad Dermatol. 2009;61(4):711-714. doi: 10.1016/j.jaad.2008.12.016. Epub 2009 Jul

5. Blaise S, Salameire D, Carpentier PH. Interstitial granulomatous dermatitis: a misdiagnosed cutaneous form of systemic lupus erythematosus? Clin Exp Dermatol. 2008;33(6):712-714. doi: 10.1111/j.1365-2230.2008.02774.x. Epub 2008 Aug 2.

6. Brecher A. Palisaded neutrophilic and granulomatous dermatitis. Dermatol Online J. 2003;9(4):1.

7. Misago $\mathrm{N}$, Inoue $\mathrm{H}$, Inoue $\mathrm{T}$, Nagasawa $\mathrm{K}$, Narisawa $\mathrm{Y}$. Palisaded neutrophilic granulomatous dermatitis in systemic lupus erythematosus with a butterfly rash-like lesion. Eur J Dermatol. 2010;20(1):128-129. doi: 10.1684/ejd.2010.0822.Epub 2009 0ct 12.

8. Misago N, Narisawa Y, Tada Y, Nagasawa K. Palisaded neutrophilic granulomatous dermatitis caused by cellulitis in a patient with systemic lupus erythematosus. Int J Dermatol. 2011;50(12):15831585.

9. Obermoser G, Zelger B, Zangerle R, Sepp N. Extravascular necrotizing palisaded granulomas as the presenting skin sign of systemic lupus erythematosus. Br J Dermatol. 2002;147(2):371-374.

10. Asahina A, Fujita H, Fukunaga Y, Kenmochi Y, Ikenaka T, Mitomi H Early lesion of palisaded neutrophilic granulomatous dermatitis in ulcerative colitis. Eur J Dermatol. 2007;17(3):234-237.

11. Hantash BM, Chiang D, Kohler S, Fiorentino D. Palisaded neutrophilic and granulomatous dermatitis associated with limited systemic sclerosis. J Am Acad Dermatol. 2008;58(4):661-664.

12. Kim SK, Park CK, Park YW, Jun JB, Yoo DH, Bae SC. Palisaded neutrophilic granulomatous dermatitis presenting as an unusual skin manifestation in a patient with Behçet's disease. Scand J Rheumatol. 2005;34(4):324-327.

13. Szepetiuk G, Lesuisse M, Piérard GE, Quatresooz P, Piérard
Franchimont C. Autoimmunity-related granulomatous dermatitis in association with hepatitis. Case Rep Dermatol. 2012;4(1):8084.

14. Mahmoodi M, Ahmad A, Bansal C, Cusack CA. Palisaded neutrophilic and granulomatous dermatitis in association with sarcoidosis. J Cutan Pathol. 2011;38(4):365-368. doi: 10.1111/j.16000560.2010.01560.x

15. Misago N, Shinoda Y, Tago M, Narisawa Y. Palisaded neutrophilic granulomatous dermatitis with leukocytoclastic vasculitis in a patient without any underlying systemic disease detected to date. J Cutan Pathol. 2010;37(10):1092-1097. doi:10.1111/j.16000560.2009.01466.x. Epub 2009Nov 9.

16. Rosenbach M, English JC 3rd. Reactive Granulomatous Dermatitis: A Review of Palisaded Neutrophilic and Granulomatous Dermatitis, Interstitial Granulomatous Dermatitis, Interstitial Granulomatous Drug Reaction, and a Proposed Reclassification. Dermatol Clin. 2015;33(3):373-387. doi: 10.1016/j.det.2015.03.005. Epub 2015 Jun 5.

17. Finan MC, Winkelmann RK. The cutaneous extravascular necrotizing granuloma (Churg-Strauss granuloma) and systemic disease: a review of 27 cases. Medicine. 1983;62(3):142-158.

18. Hau E, Vignon Pennamen MD, Battistella M, Saussine A, Bergis M, Cavelier-Balloy B, et al. Neutrophilic skin lesions in autoimmune connective tissue diseases: nine cases and a literaturereview. Medicine (Baltimore). 2014;93(29):e346. doi:10.1097/ MD.0000000000000346

19. Ne'meth T, Mo'csai A. The role of neutrophils in autoimmune diseases. Immunol Lett. 2012;143(1):9-19. doi:10.1016/j.imlet.2012.01.013. Epub 2012 Feb 11.

20. Caproni M, Torchia D, Cardinali C, Volpi W, Del Bianco E, D’Agata A, et al. Infiltrating cells, related cytokines and chemokine receptors in lesional skin of patients with dermatomyositis. Br J Dermatol. 2004;151(4):784-791. 